\title{
Médiévales
}

Langues, Textes, Histoire

47 | automne 2004

Îles du Moyen Âge

\section{Le patronage architectural du pape Pie II Piccolomini à Sienne}

The Architectural Patronage of Pius II Piccolomini in Siena

\section{Fabrizio Nevola}

\section{(2) OpenEdition \\ 1 Journals}

\section{Édition électronique}

URL : https://journals.openedition.org/medievales/498

DOI : 10.4000/medievales.498

ISSN : 1777-5892

Éditeur

Presses universitaires de Vincennes

\section{Édition imprimée}

Date de publication : 1 décembre 2004

Pagination : 139-152

ISBN : 2-84292-157-7

ISSN : 0751-2708

\section{Référence électronique}

Fabrizio Nevola, «Le patronage architectural du pape Pie II Piccolomini à Sienne », Médiévales [En ligne], 47 | automne 2004, mis en ligne le 02 septembre 2006, consulté le 23 avril 2022. URL : http:// journals.openedition.org/medievales/498; DOI : https://doi.org/10.4000/medievales.498

Ce document a été généré automatiquement le 23 avril 2022.

Tous droits réservés 


\title{
Le patronage architectural du pape Pie II Piccolomini à Sienne*
}

\author{
The Architectural Patronage of Pius II Piccolomini in Siena
}

Fabrizio Nevola

1 Enea Silvio Piccolomini naquit à Corsignano (devenu Pienza) le 18 octobre 1405. Il étudia au Studium de Sienne dès l'âge de 18 ans et entreprit une carrière de secrétaire et d'humaniste, notamment au service de l'empereur Frédéric III. En 1447 il entra dans les ordres et fut immédiatement nommé évêque de Trieste par Eugène IV. Trois ans plus tard, en 1450 il monta sur le siège épiscopal de Sienne et devint cardinal en $1456^{1}$. Durant son épiscopat siennois, Enea Silvio prépara la rencontre de Frédéric III avec Éléonore de Portugal, près des murs de la ville. Par la suite, il se rendit avec le couple à Rome où il assista au couronnement impérial de Frédéric et à son mariage avec Éléonore $^{2}$. Enea Silvio décrit ces événements dans ses Commentaires, rapportant que, durant le voyage vers Rome, ayant atteint le sommet de la colline d'Acquapendente, l'empereur se tourna vers lui et lui prédit son accession au cardinalat et son élection au siège de saint Pierre.

2 Au cours de son pontificat, Enea Silvio déploya des contacts diplomatiques avec le régime populaire de la Commune de Sienne pour obtenir le retour des familles de magnats et de nobles siennois au gouvernement de l'État, ainsi que la récupération de leurs droits. Son initiative était clairement intéressée puisque les Piccolomini avaient été exclus du gouvernement par les lois «antimagnatizie » de 1277, et qu'un certain nombre de membres de la famille associés à Enea Silvio avaient participé à un coup d'État manqué en 1450, à la suite duquel ils avaient été exilés. Dès septembre 1458, la branche de Corsignano des Piccolomini fut ainsi admise au «Monte del Popolo », un stratagème qui permit au gouvernement siennois d'éviter la publication d'un acte de réadmission générale de tous les "Gentiluomini». Cette décision engendra une situation singulière puisque désormais une des familles de la plus ancienne aristocratie siennoise devint une des plus influentes du « Popolo » ${ }^{3}$.

3 Bien que la Commune n'ait pas satisfait toutes les requêtes politiques des Piccolomini, Pie II ne retira pas ses faveurs à sa ville familiale, comme on l'a souvent suggéré, et il 
continua à s'y rendre. En 1458, il éleva Sienne au rang d'archevêché et en juin 1461, il canonisa Catherine de Sienne tandis qu'il accordait à la République siennoise des droits sur les territoires pontificaux de Radicofani et du Monte Amiata. Maintes indulgences papales furent accordées aux églises siennoises, et comme Nicholas Adams l'a remarqué, la fortification et la restauration de Corsignano/Pienza améliorèrent sensiblement les défenses des frontières siennoises méridionales. En 1464, sur la route d'Ancône où il mourut, Pie fit don du bras de saint Jean-Baptiste à la cathédrale. En raison de l'importance de cette relique - enlevée de Saint-Pierre de Rome en grande pompe par la « Porta del Popolo » - ce don paraissait considérable. D'autant que JeanBaptiste était le saint patron de Florence, et que la donation pouvait être considérée comme un affront fait aux Florentins, qui eux ne possédaient, à cette date, aucune relique importante de leur saint. J'espère montrer que le patronage architectural de Pie II à Sienne peut être considéré comme une preuve supplémentaire de l'attitude conciliante du pontife envers le gouvernement de la ville. En effet, le patronage de Pie II à Pienza fut dépassé par les nombreux projets entrepris par les Piccolomini dans le centre même de Sienne, pour lesquels le gouvernement siennois offrit des exemptions fiscales et des concessions; ces projets servirent de précédents importants aux stratégies patronales d'autres familles siennoises durant le dernier tiers $\mathrm{du} \mathrm{xv}^{\mathrm{e}}$ siècle. De plus, il semble évident que la politique incitative menée par les édiles pour renouveler le paysage urbain - que j'ai décrite par ailleurs - a influé sur le patronage choisi par le pape Pie II et par ses parents à Sienne ${ }^{4}$.

Planification civique et patronage privé : l'exemple du Palazzo delle Papesse

Les liens entre les politiques publiques et les initiatives des Piccolomini peuvent être clairement observés dans le cas du Palazzo delle Papesse, construit pour la seconde sœur de Pie II, Caterina, à partir de 1459. Ce palais, indépendant de tout habitat voisin, fut construit sur l'actuelle " piazza Manetti », un site qui faisait face à la "via di Città " et qui était entouré de rues étroites menant à la cathédrale. Malgré la proximité du "Duomo ", le lieu était loin des principales résidences familiales des Piccolomini et fut peut-être choisi pour encourager les projets de réaménagements urbains proposés par les "Maestri sopra l'Ornato", les fonctionnaires gouvernementaux chargés de l'embellissement urbain. Les maîtres de "l'Ornato" apportèrent leur soutien à la rénovation de l'espace autour de la "piazza Manetti » et leurs encouragements furent dispensés dès la fin du mois d'avril 1459 à « quiconque veut construire sur ce site [...] embellissant cette zone qui fait honte à la ville, surtout en vue de la visite imminente de la cour pontificale $»^{5}$.

5 Au moment de l'avènement d'Enea Silvio au siège pontifical, Caterina Piccolomini était veuve de Bartolomeo Guglielmi et vivait dans la "via del Casato » avec son beau-fils Francesco di Bartolomeo Guglielmi. En raison de son veuvage, Caterina dépendait pour ses revenus de son beau-fils, comme Antonia sa fille dont la dot devait être versée à Bartolomeo della Massa. Ni Antonia ni Caterina ne reçurent les sommes totales qui leur étaient dues ${ }^{6}$. L'élection du frère de Caterina au pontificat en 1458 mit fin à leur précarité. Caterina reprit son nom de famille; il fut aussi donné à son gendre, Bartolomeo dei Piccolomini della Massa, qui devint préfet de Spolète. Les fonds pontificaux aidèrent donc Caterina à retrouver son identité et son indépendance, et lui permirent aussi de devenir la destinataire d'un grand palais à Sienne, où elle vécut avec sa fille et son gendre. Il n'est guère surprenant que son petit-fils ait été appelé Aeneas en mémoire de son généreux grand-oncle. 
6 Le projet du palais progressa rapidement pour deux raisons : d'une part, grâce aux sommes constamment versées par la «Tesoreria Segreta » du pape qui couvrirent les coûts de construction, d'autre part grâce à l'obtention d'un site adéquat, due à l'intercession de la Commune de Sienne. En octobre 1459, un pétitionnaire anonyme réclamait la concession du site de la "piazza Manetti », afin d'y bâtir rapidement un palais, dont la façade devait être achevée à la fête de l'Assomption de l'année suivante. L'auteur de la pétition était, bien sûr, Caterina qui reçut une partie du site en décembre 1459 et qui élargit l'emplacement par des acquisitions nouvelles réalisées en août 1460, obtenant de la Commune un terrain supplémentaire en octobre de la même année. Le gouvernement siennois permit aussi à Caterina d'utiliser les petites rues adjacentes afin de stocker les matériaux et de dresser les échafaudages. De plus, des exemptions de taxes furent accordées sur l'importation de matériaux de construction de luxe comme le « macigno» (« marbre travertin ») et autres pierres nécessaires. Comme le déclarait le Conseil, de telles concessions témoignaient de la déférence que la Commune entendait manifester au pape, mais étaient aussi destinées à «rendre la dite maison très honorable, au moyen de grandes dépenses et d'ajouter à la gloire de cette magnifique cité ».

7 Cette "grande dépense » fut garantie directement par les finances pontificales ${ }^{7}$. Dès juin 1459 Caterina reçut des paiements réguliers qui furent effectués durant tout le règne de Pie II. Les premiers versements furent importants, mais n'avaient aucun destination spécifique. À partir de juin 1462, la majorité des dépenses dans les comptes de la Trésorerie Secrète précisent que les fonds étaient destinés « au palais qu'elle est en train de faire bâtir ». Presque 4000 ducats furent ainsi versés à Caterina entre 1459 et 1463. En plus de ces fonds, le gendre de Caterina, Bartolomeo della Massa, reçut un salaire considérable qu'il dépensa pour les travaux. À la mort de Pie II, la rapide construction du palais, entièrement dépendante du financement pontifical, s'arrêta. Comme le précisent Caterina et son gendre dans leur déclaration d'impôts de 1466, «ils subirent des dommages à l'issue de la mort du Pape Pie II parce qu'ils avaient laissé 3000 ducats à Spolète». Aussi pour continuer la construction du palais pour «l'honneur et la beauté de la ville» ils durent vendre une grande partie de leur patrimoine pour rassembler les fonds nécessaires ${ }^{8}$. Les finitions restèrent incomplètes et les dépenses d'achèvement furent estimées à 2000 florins dans la déclaration d'impôts qui fut établie au xvI siècle.

8 En utilisant des fonds de la Chambre apostolique, Pie II assista considérablement sa sœur dans la construction de son palais, mais il soutint aussi indirectement la politique du gouvernement siennois qui demandait la réorganisation de la zone de la «piazza Manetti », et plus généralement l'amélioration des façades le long de la route de Rome. De fait, dès 1444 le site de la «piazza Manetti » avait été l'objet de restructurations, et Nanni Marsili avait été sommé de restaurer le palais Marsili, qui s'écroulait. Après avoir été un premier temps exproprié, en avril 1459, Nanni Marsili put récupérer ses biens, et commissionna Luca di Bartolo da Bagnocavallo pour restaurer le palais. Ce fut à ce moment, comme nous l'avons vu, que l'office de "l'Ornato" se mit à chercher un propriétaire qui développerait le site, une offre qui fut acceptée par Caterina avec le soutien financier de son frère.

9 En sorte que, le patronage Piccolomini sur le site de la "piazza Manetti » participa activement au processus de renouvellement urbain favorisé par le gouvernement, et constitua un exemple pour les voisins. Ainsi, non seulement le palais Marsili fut 
remodelé, mais d'autres suivirent aussi. Un peu plus loin que le palais Marsili, se trouvait la propriété de la famille Lolli. Goro di Niccolò Lolli était un des plus anciens amis de Pie et il le servit en tant que secrétaire personnel. En reconnaissance de son zèle et de son amitié, Pie II accorda à sa famille le droit d'utiliser le nom Piccolomini. Dans sa déclaration d'impôts de 1465 Goro déclarait qu'il avait restauré la façade du palais "durant les jours heureux [de Pie] d'une manière magnifique», et qu'il avait besoin encore de 1200 florins pour compléter les travaux intérieurs. D'importantes armoiries des Piccolomini surmontaient le portail en commémoration de l'association entre la famille et le pape. De plus, la démolition de galeries de bois («ballatoi ») le long de la "via di Città ", réalisées par l'office de "l'Ornato", servit aussi à améliorer l'apparence générale de la zone, qui était la route principale, empruntée par les processions cérémonielles entre le Dôme et le « Campo ». Le palais de Caterina finit par être connu sous le nom de Palazzo delle Papesse. En effet, le bâtiment finit par représenter une lignée féminine, associée au nom papal des Piccolomini. Toutefois, l'importance des investissements et les ambitions architecturales exprimées dans l'autre projet familial de Pie II, qui fut développé autour de la place Piccolomini, près de San Martino, révèle à quel point les espérances du pape étaient encore plus grandioses pour les quatre fils de son autre sœur, Laudomia Todeschini Piccolomini.

Famille et mémoire : la réorganisation de la « piazza Piccolomini »

10 Enea Silvio Piccolomini est né en exil, son grand-père ayant été banni de la ville en 1368 , et son père ayant été initialement employé en tant que courtisan et mercenaire de Gian-Galeazzo Visconti, pour revenir s'installer par la suite à Corsignano aux marges du territoire siennois9. Cette branche des Piccolomini avait donc été chassée de Sienne à partir du XIV $v^{e}$ siècle; une situation à laquelle Pie II semble avoir consciemment cherché à remédier à travers ses interventions politiques et ses investissements mobiliers. Après un si long discrédit, cette branche ne possédait plus aucune propriété dans la ville, en conséquence leur présence physique et légale y était effacée.

11 Le désir du pape de rendre à sa lignée une position prééminente dans la vie politique siennoise, et de lui assurer une présence architecturale appropriée dans la ville, fut partagé entre ses deux sœurs, Laudomia et Caterina. Alors que Caterina n'avait qu'une fille, Laudomia était mariée à Giovanni Todeschini et avait quatre fils, auxquels le pape accorda des titres importants, leur assurant une position sociale de premier plan: Francesco devint archevêque de Sienne puis cardinal, Antonio, duc d'Amalfi, Giacomo, seigneur de Montemarciano et Andrea seigneur de Castiglion della Pescaia et de l'île del Giglio $^{10}$. Ce fut la branche Todeschini Piccolomini qui fut admise au « Monte del Popolo ", et qui devint active dans les offices gouvernementaux durant la seconde moitié $\mathrm{du} \mathrm{Xv}^{\mathrm{e}}$ siècle, bénéficiant d'alliances matrimoniales avec des familles siennoises ou étrangères. Alors que le palais de Caterina ancrait sa lignée au cœur de la ville, Laudomia et ses fils, qui bénéficièrent eux-aussi des subsides du pape, érigèrent une demeure monumentale dans l'enclave Piccolomini adjacente au «Campo». Ainsi les manœuvres politiques de Pie II placèrent sa branche familiale dans une position dominante au sein du puissant "Monte del Popolo", alors que ses ambitions architecturales redéfinissaient le clan Piccolomini autour du pouvoir dominant de ses neveux.

12 Dans une lettre envoyée de Rome, en septembre 1458, par l'ambassadeur siennois auprès du pape, Niccolò Piccolomini rapporta que Pie II avait dit : « je veux construire un palais chez les Piccolomini » (« in casa ai Piccolomini ») ${ }^{11}$. La lettre contenait aussi 
les remerciements formels du pape au gouvernement siennois pour avoir admis sa famille au sein du groupe dirigeant. Un lien fut donc établi dès le début entre les programmes politiques, familiaux et architecturaux qui furent résumés dans le projet de réorganisation du site de la « piazza Piccolomini ».

La "piazza Piccolomini » constituait un espace public au sud du Campo, situé entre la route de Rome et la "via del Porrione »; elle était délimitée par une série de résidences et la façade de l'église San Martino. Le grand nombre de propriétés de la famille Piccolomini autour de la place et la présence d'un puits, connu comme le «pozzo dei Piccolomini » avait conduit à nommer cet espace "piazza Piccolomini ", dès le XIV siècle. En 1453, avant même l'élection de Pie II, seize des dix-neuf foyers qui composaient la famille Piccolomini étaient taxés dans le district de Pantaneto, dans le voisinage de la place. Cette situation perdura. En 1481, quatorze foyers Piccolomini continuaient à vivre à Pantaneto, même si, à cette date onze foyers étaient taxés en dehors du district. Ce fut cette zone que Pie II sélectionna pour son patronage architectural le plus important à Sienne, car elle constituait le cœur de l'espace familial, et de surcroît, elle était placée de manière prédominante au centre de la ville, tout proche du « Campo » et de la "Strada Romana ».

"piazza Piccolomini » passa par la construction de nouveaux et vastes bâtiments, et d'une « loggia », placés de façon à réorienter l'espace en direction de la route de Rome ${ }^{12}$. La première phase de ces aménagements précéda l'élection pontificale, puisque nous savons qu'Enea Silvio paya la restauration de l'église San Martino alors qu'il était encore évêque de Sienne; le nouvel édifice fut consacré le 10 août 1458. San Martino était une église paroissiale, mais elle donnait aussi son nom au district ou Tiers de la ville qui s'étendait au sud-est du « Campo » vers la « Porta Romana ». Les Piccolomini formaient la plus puissante famille résidante dans ce district et participaient fréquemment au gouvernement local. Le bâtiment fut reconstruit au $\mathrm{XvI}^{\mathrm{e}}$ siècle et aucune trace n'a survécu de la rénovation du Xve siècle.

Alors que Niccolò Piccolomini rapporte la volonté précoce du pape, dès son avènement, de construire un palais dans le quartier Piccolomini, le projet n'aboutit pas immédiatement; il fut précédé par la construction d'une magnifique "loggia » en marbre. Il semble désormais assuré que l'église San Martino faisait partie intégrante du projet de réaménagement de la "piazza Piccolomini », puisque le plan originel de la «loggia " l'alignait sur la façade de San Martino. Cependant, ceci aurait nécessité l'utilisation d'un terrain appartenant à Giovanni di Guccino, un maître d'école qui refusait de vendre son bien aux Piccolomini. Les plans furent donc modifiés et la «loggia » fut construite sur un site payé par les fonds pontificaux ; 3000 ducats furent versés entre décembre 1460 et octobre 1461 pour acquérir le site et peut-être les matériaux. La campagne de construction ne commença sérieusement qu'avec un paiement de 600 ducats fin avril 1462.

16 L'auteur d'une chronique siennoise, Allegretto Allegretti, mentionne que la première colonne de la «loggia » fut érigée le 18 mai 1462, et il est probable que les travaux étaient achevés dès septembre de la même année, quand l'artiste Lorenzo di Pietro dit « il Vecchietta » et un maçon nommé Nanni Castori furent appelés pour donner leur avis sur le coût total du projet. À travers les paiements effectués par la trésorerie pontificale, il est possible d'évaluer les dépenses à près de 3811 ducats. Il est probable que ces chiffres sont complets et précis, dans la mesure où ils incluent le paiement de vingt livres en novembre 1462 pour 6 paires de chausses achetées pour les 
constructeurs "qui ont travaillé sur la loggia des Piccolomini », dernière et modeste dépense attestée pour l'édifice ${ }^{13}$. On peut donc suggérer que Pie II a pu l'admirer pendant sa brève visite à Sienne, lors de son retour de la station thermale de Bagno di Petriolo, le 31 août 1462.

La «loggia » est composée d'une arcade à trois baies, orientée au nord, le long de la "Strada Romana »; elle est construite avec des colonnes et des chapiteaux de marbre à l'antique qui soutiennent les armoiries pontificales des Piccolomini; sur l'inscription en bronze figure PIE II PONTIFEX MAXIMUS GENTILIBUS SUIS PICCOLOMINEIS. Un banc simple, articulé par des pilastres classiques court à l'intérieur de la "loggia ». L'extérieur du banc face à la "Strada Romana ", particulièrement visible parce qu'à hauteur des yeux des passants, était décoré d'une série d'armoiries sculptées, jadis peintes, appartenant à d'autres membres de la famille Piccolomini. Elles signalaient de manière évidente les liens entre Pie II et ses plus proches parents. Les Gentilibus Suis que Pie associait à son nom étaient en particulier les fils de sa sœur Laudomia, les bénéficiaires de ses plus grandes faveurs, mais aussi le reste du clan résidant dans le quartier, dont les armes sont visibles sur les coins de la mansarde face à la route et à la place.

Alors qu'Antonio et Francesco firent leur carrière respectivement à la cour napolitaine et à la Curie, leurs frères Andrea et Giacomo restèrent à Sienne. Ces derniers héritèrent de la « loggia ». Le bâtiment était entretenu à leurs frais, en partie à l'aide des revenus des magasins situés dans le sous-sol. Ils étaient aussi propriétaires de l'imposant palais Piccolomini, qui dominait l'espace entre la "piazza Piccolomini » et le "Campo », avec sa façade sur la "Strada Romana ». Comme nous l'avons observé, le désir du pape de construire un palais au cœur de l'enclave des Piccolomini fut exprimé dès ses premiers échanges avec la Commune de Sienne. Toutefois, deux années s'écoulèrent, et trois visites à Sienne s'effectuèrent avant qu'une action n'ait été entreprise. L'attention du pape s'était plus précisément focalisée sur Pienza et le projet de la « loggia » à Sienne. L'emplacement et le début du chantier du palais sont mentionnés pour la première fois dans une requête adressée en octobre 1460 par l'agent de Pie II à Sienne, Giovanni Saracini. Il demandait une exemption de la moitié des impôts fonciers ("gabelle dei contratti») et un allègement des taxes d'importation de matériaux de construction. Cela fut accordé parce que «la ville sera rendue plus belle et magnifique grâce à ce projet merveilleux ». Dès le début, le projet du palais fut donc présenté comme une contribution à l'amélioration de l'image publique de la ville.

L'acquisition du site commença une fois les exemptions accordées, et le pape dépensa la somme de 8300 ducats (entre janvier 1461 et octobre 1463) ${ }^{14}$. L'essentiel de cette somme - environ 6000 ducats - fut payé en mai 1461 ; la part restante fut employée à l'acquisition de quelques terrains nécessaires pour compléter le site. On ne sait si d'autres propriétés furent acquises après 1463 , mais l'emplacement devait être presque entièrement aux mains des Piccolomini en 1464, puisque les 16000 ducats que Pie II laissa au cardinal Francesco étaient destinés «à construire le palais » ${ }^{15}$. Malheureusement, comme les neveux du pape s'en plaignaient en 1466, le testament ne fut pas respecté et les fonds assignés furent bloqués par le nouveau souverain pontife, Paul II. Ils protestèrent faisant valoir que « pour que notre palais soit sur le sol, [... ] pour notre propre honneur et celui de la ville [...] puisqu'il n'est pas encore achevé [...] cela nous coûtera de nombreux milliers de florins ». 
20 Dans son état actuel, le palais Piccolomini est incomplet, puisqu'il devait occuper seul une « insula » entière entre le "Campo », la "Strada Romana », la « via del Porrione » et la "piazza Piccolomini », et disposer de quatre façades identiques à la seule qui fut construite. En 1480, le palais fut divisé en deux parties ${ }^{16}$. L'une d'entre elles appartenait à Giacomo. Elle est connue sous le nom de «Palazzo Nuovo" (le nouveau palais) et faisait face à la "Strada Romana ». Elle était construite en travertin, dans un style similaire à celui adopté pour le palais de Pienza. L'autre, que détenait Andrea et qui était décrite dans les documents comme le «Casamento Vecchio» (l'ancienne résidence), donnait sur un angle du «Campo » et disposait d'une façade sur l'étroite «via del Porrione»; elle avait une structure plus complexe, formée de nombreux édifices antérieurs dont l'apparence fut unifiée grâce à l'insertion de nouveaux châssis aux fenêtres et d'autres détails architecturaux.

21 En parvenant à entreprendre ce palais au cœur de l'espace du clan des Piccolomini, Pie II paraît avoir fait mentir l'usage selon lequel les conflits familiaux tournent généralement autour de propriétés et empêchent tous travaux. Toutefois, en cherchant à former un emplacement assez large pour établir un bâtiment cubique et massif, ses ambitions semblent avoir échoué face à la réticence de certains membres du clan à lui céder leur propriété. Dans le document qui établit le partage du palais en deux, le «Casamento Vecchio» est décrit comme étant clôturé du côté de Porrione-« piazza Piccolomini » par une série de propriétés appartenant à d'autres membres du clan. Ces propriétaires empêchèrent l'achèvement du palais selon le plan initial et les conflits qu'ils suscitèrent durèrent jusqu'au XVI ${ }^{\mathrm{e}}$ siècle.

Cette attitude suggère combien Pie II situait les besoins et les ambitions de ses parents immédiats en relation avec ceux de la totalité du clan Piccolomini. Dans une certaine mesure la défense des intérêts des Piccolomini, dans leur ensemble, constitua un obstacle à la promotion de sa propre branche. Par la suite aucun membre de la famille, sauf ceux qui étaient liés directement au pape, ne payèrent de charges fiscales pour la «loggia "; même s'il est dit que Pie établit une "consorteria » pour contrôler les propriétés communes des Piccolomini, aucune déclaration d'impôts conjointe ne fut effectuée pour des biens possédés en commun dans la ville ${ }^{17}$. Il n'en est pas de même à Florence, où la « loggia » construite durant la même période par les Ruccellai est une «loggia commune", payée à partir de fonds communs, comme l'a montré Francis Kent $^{18}$.

23 Toutefois, il serait erroné de croire que seuls les Piccolomini «papeschi » - nom sous lequel ils étaient parfois connus - furent les bénéficiaires de la munificence pontificale. De nombreuses armoiries appartenant à d'autres branches de la famille placées autour de la place proclament fièrement leur association à la lignée papale. Comme le montre un dessin du $\mathrm{xvII}^{\mathrm{e}}$ siècle de la collection Chigi au Vatican, une importante tour médiévale faisait face au complexe "loggia-piazza»; elle reliait les nouveaux bâtiments au reste des possessions familiales, plus anciennes, de la zone. Par la disposition des emblèmes familiaux et l'occupation commune du site le vaste clan paraissait uni, alors que le style architectural de leurs biens distinguait les neveux du pape, les Todeschini Piccolomini de leurs parents.

Architecture des palais

24 Les réalisations architecturales entreprises par le pape traduisaient l'importance de son patronage, tant par leur taille et leur coût, que par leur forme innovante. La " loggia » et les deux palais Piccolomini marquent un moment nouveau dans l'histoire 
architecturale de Sienne, l'introduction du style «all' antica». Cette nouvelle forme bien distincte souligne les liens des Piccolomini avec la Rome pontificale, et intègre la famille parmi les élites raffinées qui choisissent de plus en plus un modèle classique pour leur palais. À leur tour, ces projets servirent de modèles et inspirèrent d'autres " patrons » siennois, qui construisirent leur palais plus tard ${ }^{19}$.

Malgré l'indéniable importance des palais, leur paternité reste incertaine: à quel architecte ou quel groupe d'architectes faut-il attribuer les travaux? Le problème est bien illustré par le conflit qui émergea en juin 1463, concernant le salaire que Caterina Piccolomini devait payer pour le palais «delle Papesse » : un arbitrage fut demandé à un certain maître Bernardo - généralement identifié avec Bernardo Rossellino - pour une somme qui était due à Antonio Federighi. Deux ans plus tard, Caterina lui devait toujours 400 florins. En janvier 1473, elle comparaissait devant la cour de la "Mercanzia " pour un litige qui l'opposait au sculpteur et maçon Urbano di Pietro da Cortona, au sujet « de fenêtres de marbre et du prix de deux Madonnes [...] ». Le juge des prix, Pietro dell'Abaco, assigna à Urbano 100 livres de réparation. Les architectessculpteurs Bernardo Rossellino et Antonio Federighi, le sculpteur-maçon Urbano da Cortona et l'expert Pietro dell'Abaco sont tous mentionnés en association avec le bâtiment, mais aucun d'entre eux n'est fermement identifié comme étant son architecte.

Le bâtiment même nous fournit peu d'indices pour résoudre cette question d'attribution. La façade du « palazzo delle Papesse » trouve son parallèle le plus proche dans le palais Medici-Riccardi à Florence; les édifices partagent la même gradation dans l'élévation: un bossage massif au rez-de-chaussée, des pierres de taille avec des cannelures aux étages supérieurs. De même l'arc légèrement brisé des fenêtres renvoie aux formes traditionnelles des propriétés voisines, tout comme à Florence, les fenêtres du palais Médicis ressemblent à celles du «Palazzo Vecchio » ainsi que l'a montré Howard Burns. Pareillement, les arcs en plein-cintre, qui formaient autrefois une « loggia " au rez-de-chaussée, rappellent le modèle médicéen, mais ils peuvent aussi faire référence au palais Tolomei datant $\mathrm{du}$ XIII ${ }^{\mathrm{e}}$ siècle qui contenait aussi au rez-dechaussée une «loggia» symétriquement ordonnée. Le respect des traditions de construction locales siennoises est encore plus évident à l'intérieur des bâtiments : dans la cour, des colonnes octogonales en briques soutiennent des arcs en plein-cintres avec des enchaînements de pilastres scandant l'entière élévation du bâtiment, font référence aux dispositions du "Cortile del Podestà » du « Palazzo Pubblico ». Alors que le palais Medici de Florence influença le dessin de l'édifice, l'exécution du projet, comme les détails de construction, marquent le poids considérable des traditions architecturales ou ornementales locales ${ }^{20}$.

Plusieurs noms paraissent liés au projet de la «loggia » Piccolomini. En mars 1460, un premier dessin, accompagné d'un modèle en bois, fut soumis par Lorenzo di Pietro, dit « Il Vecchietta ». Charles Mack et Ruth Rubinstein ont suggéré que Bernardo Rossellino aurait pu dessiner la bâtiment. Plus récemment, Lawrence Jenkins a proposé le nom improbable de Leon Battista Alberti. Néanmoins, une série de documents montrent que Antonio Federighi supervisa la construction et qu'il aurait pu en être le sculpteur principal. De plus, d'importantes similitudes entre les sculptures des chapiteaux et des blasons de la «loggia ", avec celle de la «Mercanzia », où la participation d'Antonio Federighi est attestée, confirment son rôle dominant dans le projet. 

mmissionnés à Sienne, l'engageant pour sculpter la tombe de ses parents à San Francesco en 1459, et à un niveau ou un autre dans tous ses projets architecturaux. Parmi les premiers paiements enregistrés par la Chambre apostolique en 1459, 276 florins caméraux furent donnés à "Antonio Federighi maitre de l'œEuvre de la cathédrale de Sienne » pour la restauration des chambres qui devaient être utilisées " comme résidence de notre Saint Père " ${ }^{21}$. L'œuvre de Federighi témoigne d'une connaissance considérable de l'architecture et de la sculpture classiques, et nous savons qu'il se rendit à Rome pour étudier en 1448 ou 1450. Inversement, que Bernardo Rossellino tout comme le Vecchietta aient été appelés en tant qu'arbitres des projets de construction des Piccolomini auxquels Antonio Federighi était associé (la «loggia » et le palais delle Papesse) suggère qu'ils n'étaient pas employés directement dans ces projets, et rend encore plus crédible le fait qu'Antonio en ait été le superviseur. Ce dernier aura donc probablement œuvré comme le véritable architecte, exécutant plusieurs bâtiments des Piccolomini à Sienne, même si leurs plans résultaient d'un 
travail complexe mené entre le commanditaire, le maître d'œuvre et peut-être même un conseiller extérieur, tel Bernardo Rossellino.

Quel qu'ait été l'architecte des trois bâtiments majeurs financés par la chambre apostolique pour les Piccolomini à Sienne, les choix stylistiques, s'inspirant délibérément de modèles non-siennois, montrent que ces projets étaient liés à celui de Pienza. Style architectural particulier et conception des bâtiments tenant compte de leur intégration au tissu urbain réunissent certainement les deux entreprises. Cependant, à Sienne, Pie II imposa un rythme différent de celui qui gouvernait ses choix à Pienza. De même, le fait qu'il ait investi dans le palais de Caterina, loin de la "piazza Piccolomini», suggère que ses initiatives n'étaient pas exclusivement réservées à un espace urbain particulier et qu'il espérait participer aux projets de renouvellement de la commune.

Par contraste, la réalisation de la loggia et du palais Piccolomini était le moyen de fournir à ses parents les plus proches une résidence placée de manière dominante dans l'espace du clan Piccolomini. Dans tous les cas, le choix d'un style architectural nouveau singularisait les bâtiments et surtout leurs propriétaires désormais considérés comme des individus raffinés, entretenant de bonnes relations avec une élite culturelle italienne étendue. Alors que les modèles de la Rome pontificale et ceux dispensés par les élites florentines ont peut-être inspiré les plans des deux palais et de la «loggia », ces projets s'intègrent cependant pleinement au contexte politique et urbain siennois.

\section{NOTES}

*. Ce texte est issu d'une communication prononcée le 7 mai 2003 au musée du Petit Palais d'Avignon à l'invitation de Didier Boisseuil et d'Esther Moensch. J'ai choisi - pour des raisons de place - de maintenir l'essentiel du texte présenté et de limiter l'importance des notes infrapaginales; pour de plus amples précisions, je renvoie le lecteur au chapitre 2 de mon livre à paraitre Architecture and Government in Renaissance Siena. Fashioning Urban Experience (1400-1555). Je tiens à remercier Emma del Balzo di Prese et Didier Boisseuil pour l'aide qu'ils m'ont apportée lors de la traduction.

1. L. Totaro, Pio II nei suoi Commentarii, Bologne, 1978, p. 29-88.

2. F. NEVOLA, « "Lieto e trionfante per la città" : Experiencing a mid-fifteenth-century imperial triumph along Siena's Strada Romana », Renaissance Studies, 17-4, 2003, p. 581-606.

3.Cf. I. Polverini FosI, « La Comune Dolcissima Patria : Pio II e Siena », dans D. RUGiadinI éd., I ceti dirigenti nella Toscana del Quattrocento, (Atti del V e VI convegno : Firenze, 10-11 dicembre 1982 ; 2-3 dicembre 1983), Florence, 1987, p. 509-21 ; P. PERTICI, « Il viaggio del papa attraverso il territorio senese : le tappe di una vita » dans Il sogno di Pio II e il viaggio da Roma a Mantova. Atti del Convegno Internazionale. Mantova : 13-15 aprile 2000. (Centro Studi L. B. Alberti, Ingenium n. 5), A. Calzona, F. P. Fiore, A. Tenenti and C. Vasoli dir., Florence, 2003, 143-62. 
4.F. NeVolA, «"Ornato della città" : Siena's Strada Romana as Focus of Fifteenth-century Urban Renewal », Art Bulletin, 82-1, 2000, p. 26-50.

5.Archivio di Stato di Siena (désormais ASS), Consiglio Generale 228, fo 168 , le 4 avril 1459 (cité dans P. Pertici, La città magnificata : interventi edilizi a Siena nel Quattrocento, Sienne, 1995, p. 66).

6. La restitution de dot est mentionnée dans ASS, Lira 136, fo 13 (1453) et Lira 156, fo 19 (1466) ; les détails biographiques sont dans C. Ugurgeri della Berardenga, Pio II Piccolomini, con notizie su Pio II e altri, Florence, 1973, p. 135, p. 220.

7. Largement documentée dans Archivio di Stato di Roma (désormais ASR), Camerale Primo, Mandati Camerali 834-6 et Tesoreria Segreta 1288-9.

8. ASS, Lira 156, fo 19 (1466).

9. C. Ugurgeri della Berardenga, op. cit., p. 28-34.

10. Cf. les brèves remarques dans C. Ugurgeri della Berardenga, op. cit., p. 504-545; et

G. A. Campano, « Vita Pii II Pontificis Maximi » dans RIS, III.3, G. Zimolo éd., Bologne, 1964, p. 66.

11. ASS, Concistoro 1992, fo 77 (le 27 septembre 1458).

12. A. Lawrence Jenkens, « Pius II's Nephews and the Politics of Architecture at the End of the Fifteenth Century in Siena », Bullettino Senese di Storia Patria, CVI, 1999 [2001], p. 68-114; une lecture plus nuancée se trouve dans F. Paolo Fiore, "Siena e Urbino », dans Storia dell'architettura italiana : il Quattrocento, F. Paolo Fiore dir., Milan, 1998, p. 277-279 et F. Paolo Fiore, « La Loggia di Pio II per i Piccolomini a Siena », dans Il sogno di Pio II, op. cit., p. 129-142.

13. ASR, Tesoreria Segreta, 1289, fo 133.

14. ASR, Tesoreria Segreta 1288, fo 74 (le 8 janvier 1461) et les entrées suivantes aux fos $75,79,101,108 \mathrm{v}^{\circ}, 118$.

15. ASS, Lira 166, fos 12-13. Pour la campagne menée par Paul II contre les assignations réalisées par Pie II, cf. A. de Vincentis, Battaglie di Memoria. Gruppi, intellettuali, testi e la discontinuità del potere papale alla metà del Quattrocento, Rome 2002.

16. ASS, Consorteria Piccolomini 19 (le 9 octobre 1480); et aussi dans Consorteria Piccolomini 17, fo 53v sq. (Contratti di Andrea di Nanni Piccolomini [1464-1519]).

17. Pour la formation de la « consorteria », cf. L'archivio diocesano di Pienza, G. Chironi éd., Sienne, 2000, p. 19-25.

18. F. W. KENT, «The Rucellai Family and its Loggia », Journal of the Warburg and Courtauld Institutes, 35, 1972, p. 397-401.

19. La question de l'impact architectural et urbain des projets réalisés à Sienne grâce au patronage des Piccolomini est examinée dans mon Architecture and Government in Renaissance Siena.

20. Sur la tradition de l'architecture siennoise du Quattrocento, $c f$. H. Burns, « Restaurator de ruyne antiche : tradizione e studio dell'antico nelle attività di Francesco di Giorgio », dans Francesco di Giorgio Architetto, M. Tafuri, F. Paolo Fiore dir., Milan, 1994, p. 151-181; de même, mon « Revival or Renewal : Defining Civic Identity in Fifteenth-century Siena » dans Shaping urban identity in the middle ages, P. Stabel et M. Boone éd., Louvain/ Apeldoorn, 1999, p. 111-134, avec bibliographie.

21. ASR, Mandati camerale 834, fo 171 (le 24 juillet 1460). 


\section{RÉSUMÉS}

Cet article traite du patronage architectural de Pie II Piccolomini à Sienne, sa ville natale. Le goût sophistiqué du Piccolomini en matière d'architecture est bien connu et est illustré en particulier par les interventions monumentales qu'il commanda hors de Sienne. Nous connaissons déjà beaucoup des commandes architecturales du pape pour le Vatican, telles que la «Loggia » de la Bénédiction attribuée à Francesco dal Borgo. De même, le complexe monumental de bâtiments par lequel Bernardo Rossellino remodela le petit village de Corsignano au sud de la Toscane, le transformant en la ville de Pie-Pienza, a été maintes fois étudié. À l'opposé nous savons peu des projets du Piccolomini pour la ville de Sienne, ce qui peut paraître surprenant, d'autant que par une sorte d'ironie, l'essentiel de la documentation qui étaye cet exposé est maintenant conservé au second étage ( " piano nobile ») du palais Piccolomini, un bâtiment originellement conçu par le pape lui-même.

The Architectural Patronage of Pius II Piccolomini in Siena. This paper discusses the architectural patronage of Pius II Piccolomini in his hometown of Siena. Piccolomini's sophisticated taste in matters architectural is well known, particularly as documented in the monumental interventions he commissioned outside Siena. Much is already known of the pope's architectural commissions for the Vatican, and the monumental complex of buildings with which Bernardo Rossellino reshaped a small village in South Tuscany, transforming it into the city of Pius: Pienza. This makes it all the more surprising that we know so little about Piccolomini's projects for the city of Siena, where papal patronage prompted the construction of two monumental palaces and an « all'antica » loggia.

INDEX

Keywords : architecture, Renaissance, Patronage, Siena, Pie II

Mots-clés : Sienne

\section{AUTEUR}

\section{FABRIZIO NEVOLA}

Villa I Tatti, The Harvard University Center for Italian Renaissance Studies, Via di Vincigliata 26, I-50135 Florence 